\title{
The Expression of Galectin-3 in Tumor and Cancer-Associated Fibroblasts in Invasive Micropapillary Breast Carcinomas: Relationship with Clinicopathologic Parameters
}

\author{
(1) Yasemin Çakır ${ }^{1}$, (1D Canan Kelten Talu ${ }^{1}$, (1) Özlem Mermut², (1) Didem Can Trabulus ${ }^{3}$, (1) Esra Arslan ${ }^{4}$ \\ ${ }^{1}$ Department of Pathology, University of Health Sciences Turkey, İstanbul Training and Research Hospital, İstanbul, Turkey \\ ${ }^{2}$ Department of Radiation Oncology, University of Health Sciences Turkey, İstanbul Training and Research Hospital, İstanbul, Turkey \\ ${ }^{3}$ Department of General Surgery, University of Health Sciences Turkey, İstanbul Training and Research Hospital, İstanbul, Turkey \\ ${ }^{4}$ Department of Nuclear Medicine, University of Health Sciences Turkey, İstanbul Training and Research Hospital, İstanbul, Turkey
}

\begin{abstract}
Objective: Galectin-3 affects tumor progression and cell surface polarization by expressing from the tumor and cancer-associated fibroblasts (CAFs). Therefore, it may have a role on micropapillary carcinomas (IMPC), which have characteristic morphological features. The aim was to investigate the expression levels of Galectin-3 within tumor and peritumoral CAFs in IMPC, and to compare with expression in invasive ductal carcinomas (IDC).

Materials and Methods: Hematoxylin and Eosin-stained preparations of resection materials examined between 2010-2016 were re-evaluated. Thirtyfour IMPC cases and 34 IDC cases with similar molecular subtype distribution to IMPC were compared. Galectin-3 levels were evaluated with a calculated $\mathrm{H}$-score in tumor and semi-quantitatively in CAFs.

Results: While tumoral Galectin-3 expression levels were higher in IMPCs compared to IDCs, there was no difference for Galectin-3 expression in CAFs between the two histologic types. However, there was no significant relationship between tumoral Galectin-3 expression and clinicopathological parameters in IMPCs. When the subjects were divided into two groups, depending on their Galectin-3 status regardless of histological types, the loss of Galectin-3 expression in tumor was found to be related to larger tumor size/advanced $\mathrm{pT}$ stage and a greater number of metastatic nodes. Additionally, expression of Galectin-3 in CAFs was found to be associated with distant metastasis.

Conclusion: IMPC showed prominent Galectin-3 expression in tumor compared to IDC. However, independent from the histological type, whereas the loss of Galectin-3 expression in tumor showed an association with larger tumor size and higher number of metastatic axillary lymph nodes, the presence of Galectin-3 expression in CAFs showed an association with distant metastasis.
\end{abstract}

Keywords: Breast, cancer-associated fibroblast, galectin-3, invasive breast cancer, micropapillary carcinoma

Cite this article as: Çakır Y, Kelten Talu C, Mermut Ö, Can Trabulus D, Arslan E. The Expression of Galectin-3 in Tumor and Cancer-Associated Fibroblasts in Invasive Micropapillary Breast Carcinomas: Relationship with Clinicopathologic Parameters. Eur J Breast Health 2021; 17(4): 341-351.

\section{Key Points}

- Tumoral Galectin-3 expression was found higher in invasive micropapillary carcinomas (IMPCs) compared to invasive ductal carcinomas (IDCs).

- In IMPCs, there was no relationship between Galectin-3 expression and clinicopathological parameters.

- Independent from the histologic type of breast cancer, loss of Galectin-3 expression in tumor showed an association with larger size of tumor and a greater number of metastatic axillary lymph nodes.

\section{Introduction}

The morphological features of invasive micropapillary carcinoma (IMPC) were defined by Fisher in 1980 as an exfoliative appearance in papillary breast carcinomas (1). The term "micropapillary carcinoma” was first used by Siriankgul in 1993 for breast IMPCs (2). Since then, IMPC has been described in many locations, such as lung, bladder, and salivary glands (3).

IMPC is composed of small groups of tumor cells with no fibrovascular core that may mimic lymphovascular invasion. These groups of cells show a characteristic reverse polarization described as 'inside-out' pattern (4). In other words, the apical sides of the tumor cells are closest to the stroma rather than the luminal surface (5). This feature can be demonstrated by the presence of positive immunostaining for EMA (MUC1) on the periphery of tumor cell groups. In addition to that, microvilli observed by electron microscopic examination on the outer surface of tumor 
cell clusters support reverse polarization (6). This appearance suggests that tumor cells in micropapillary carcinoma have a higher mobility and thus are more likely to invade adjacent tissues than tumor cells in ordinary invasive breast carcinoma. Importantly, it has also been thought that microvilli-like structures can help in establishing relations with endothelial cells (4).

Cancer is a neoplastic mass in which malignant epithelial cells interact with the stromal microenvironment. This microenvironment facilitates or inhibit tumor formation and/or progression. Cancerassociated fibroblasts (CAFs) constitute the major cell group within this microenvironment (7). Based on the contribution of tumor stroma to tumor invasion and metastasis, many drugs targeting CAFs have been tried and promising results have been obtained (8). CAFs also are responsible for resistance to chemotherapeutic drugs in most tumors (8).

Galectins belong to a family of carbohydrate-binding proteins that show a high affinity for $\beta$-galactosides. Galectin- 3 , which is the only member of the chimeric galectin group, is also the most studied member of the Galectin family (9). Since Galectin-3 can demonstrate a wide distribution, both inside and outside the cell, its functions are roughly grouped into intracellular and extracellular activities (10). Intracellular functions include anti-apoptotic effect, regulation of intracellular signal transduction, gene expression, and mRNA regulation (10). Extracellular functions include the regulation of cell adhesion, angiogenesis, and immune regulation (10). All these functions associated with Galectin-3 are important features at every stage of the tumorigenesis process, from local invasion to metastasis. In recent years, it has been shown that Galectin-3 can also be expressed by CAFs, affecting all these mechanisms $(11,12)$. For this reason, Galectin-3-focused therapies are on the agenda in targeted treatment studies for a range of solid cancers, and it has been reported that it may have the potential to be a useful marker in preventing resistance to some chemotherapeutic drugs $(13,14)$.

In this study, in patients with IMPC, which exhibits an unusual morphology and organization of tumor cells and its relation to the stroma and is usually accompanied by lymphovascular invasion and axillary lymph node metastasis, we aimed to investigate: 1) Galectin-3 immunostaining properties in tumor cells and the surrounding stromal fibroblasts, and to compare them with IDC evincing a similar molecular phenotype; and 2) to reveal the relationship between Galectin-3 expression status and clinicopathological parameters and survival.

\section{Materials and Methods}

Hematoxylin and eosin (H\&E) stained sections of surgical excision materials belonging to 850 cases evaluated for breast carcinoma between 2010-2016 were retrieved from the archives of our clinic. All slides were re-examined under a light microscope. Cases containing at least a $10 \%$ micropapillary component in an invasive tumor were identified. Micropapillary morphology was confirmed by EMA (MUC1) immunohistochemical staining in all cases.

The clinical information concerning the relevant patients was gathered, either through direct contact with the managing physicians of the multidisciplinary working group and/or in some cases from the hospital's intranet system. Patients who had received neoadjuvant therapy and those with metastasis at the time of diagnosis were
In order to compare the Galectin-3 immunohistochemical staining characteristics in IMPC cases, the same number of IDC cases (nonIMPC group) with the same molecular phenotype distribution as the IMPC group were identified consecutively.

An immunohistochemical study was performed using the streptavidinbiotin method. All of the tissues were fixed with a $10 \%$ neutral buffered formalin. For each case, the tumor blocks were selected that best represented the content and histological composition of the tumor and comprised minimal necrosis and hemorrhage. Nontumoral breast parenchyma was used as the internal control. The immunohistochemical staining procedure was applied using a BenchMark ULTRA Ventana device.

For Galectin-3 (Cell Marque, 9C4 clone, 1/75 dilution), cytoplasmic and/or membranous staining in normal breast luminal epithelial cells in areas adjacent to the tumor was considered as the positive internal control. For invasive tumor cells, the staining density was evaluated as none, minimal, medium or strong and subsequently scored as $0,1,2$ or 3, respectively. (Figure 1a-d). The extent of staining was evaluated as the proportion of the stained area. As a result of multiplying these two values with each other, a value of 0 to 300 (H-score) was found where " 0 " indicated the absence of staining, and " 300 ” representing strongly intense diffuse staining across all tumor tissues. All cases except those having an $\mathrm{H}$-score value of 0 were considered positive for Galectin-3 immunohistochemical staining. For CAFs, staining intensity was evaluated as follows: $0(0 \%), 1(<10 \%), 2(10 \%-50 \%), 3(>50 \%)$ and scored ina similar fashion to Galectin-3 staining in tumor cells $(0,1,2$ or 3$)$. Group 0 was considered negative, Groups 1, 2 and 3 were considered positive (Figure 2a-b).

P53 (Ventana, DO9 clone, 1/250 dilution) immunohistochemical expression was accepted as 'positive' for nuclear staining in $50 \%$ or more of tumor cells, and as 'negative' in staining less than 50\%.

Mean \pm standard deviation, and median (minimum and maximum range) values were used as descriptive statistics to define continuous variables and frequency distribution rates, and percentages were used to describe categorical variables. Kolmogorov-Smirnov normality tests were employed to determine the normal distribution of continuous variables. Paired comparisons between groups were investigated using independent samples of t-tests and chi-square tests. Associations between variables were determined via Pearson moment correlation coefficients. Survival analysis was carried out using Kaplan-Meier and Log Rank tests. A value of $\mathrm{p}<0.05$ was accepted to indicate statistical significance. IBM SPSS, version 20 (IBM Inc., Armonk, NY, USA) was used for the analysis of all data.

Ethical approval for this study was obtained from the Ethics Committee of İstanbul Training and Research Hospital (reference no: 963).

\section{Results}

In total 34 IMPC cases were included and compared with 34 cases of IDC. The clinicopathological features of all cases are summarized in Table 1.

The Galectin-3 staining properties of the tumor cells and CAFs of the IMPC group are summarized in Table 2. The level of Galectin-3 expression in tumor cells was significantly higher in the IMPC group than in the IDC group $(\mathrm{p}<0.05)$. However, no significant difference 
was found for Galectin-3 expression level in the CAFs between the groups ( $\mathrm{p}>0.05)$ (Table 2$)$.

There was no significant association between Galectin-3 expression within the invasive tumor cells of the IMPC and other clinicopathological parameters $(p>0.05)$. However, the mean tumor size $(3.53 \pm 2.13 \mathrm{~cm})$ tended to be larger in cases with negative Galectin-3 expression in tumor cells than those with positive Galectin-3 expression (2.42 \pm 1.12 $\mathrm{cm})(\mathrm{p}=0.059)$. Additionally, the relationship between Galectin-3 expression and the pT stage approached significance $\left(\chi^{2}(2)=5.832 ; \mathrm{p}\right.$ $=0.05)$. Thus, while advanced $\mathrm{pT}$ stages were associated with the loss of Galectin-3 expression in the tumor, early pT stage was associated with the presence of Galectin-3 expression in the tumor.

All 68 cases, both IMPC and IDC, were divided into two groups, either Galectin-3 positive or negative, and the relationship between Galectin-3 expression and other clinicopathological parameters was evaluated (Table 3-4). The mean tumor size was significantly greater

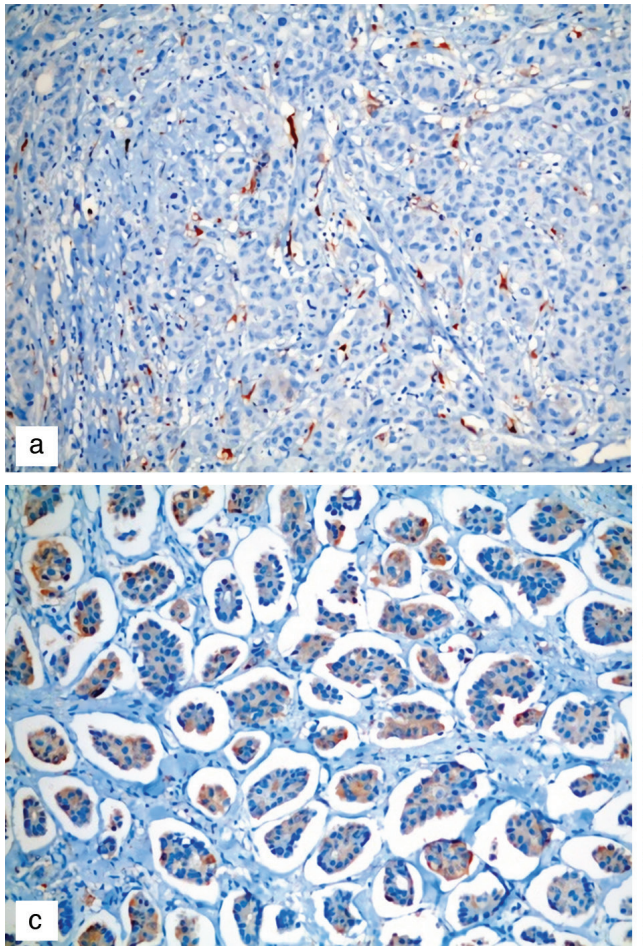

in patients with no staining for Galectin-3 in tumor cells than those with positive staining $(\mathrm{p}<0.05)$. Consistent with this, patients in the negative Galectin-3 staining group had a significantly higher pT stage and a higher number of metastatic axillary lymph nodes $(p<0.05)$. No other significant differences were identified between the positive or negative staining groups and clinicopathologic parameters (Table 3). Additionally, when patients were stratified by positive or negative Galectin-3 staining in CAFs, no significant differences in clinicopathological features were found (Table 4).

The median (range) follow-up period of all cases was 79 (1-113) months. During follow-up, $14(20.6 \%)$ patients died and distant metastases were detected in $15(22.1 \%)$ patients. The Galectin-3 staining properties in the cases with distant metastasis and/or patients who died due to breast cancer are summarized in Table 5 .

When examined with the Kaplan-Meier method, disease-free survival (DFS) and overall survival (OS) rates were higher in patients with

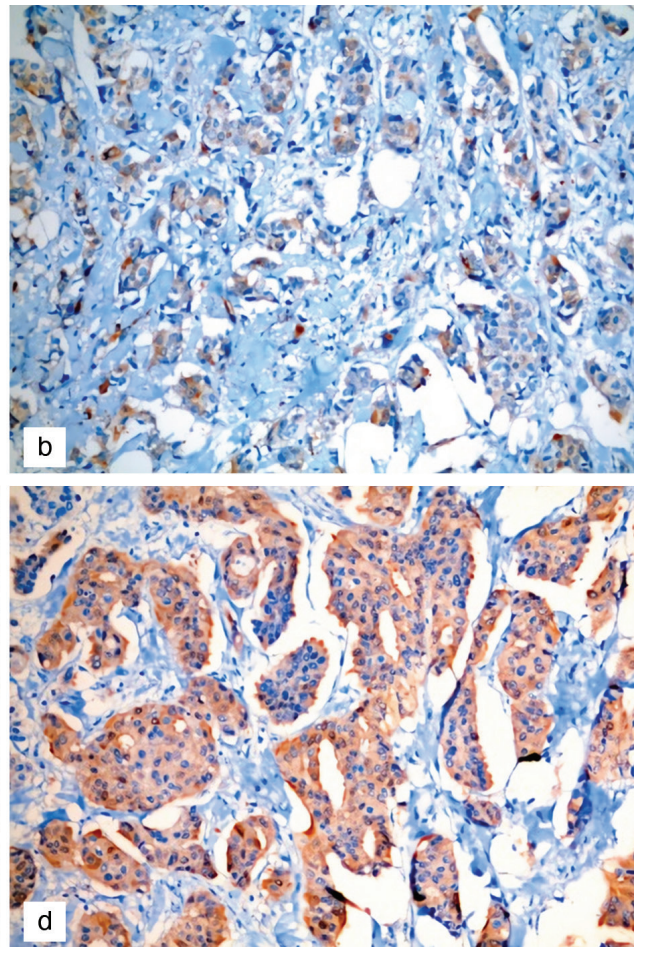

Figure 1. Immunostaining for Galectin-3 in tumor cells; a) Invasive tumor cells (Invasive ductal carcinoma) showing negative immunostaining for Galectin-3 (Score 0). Some of the inflammatory cells were seen as positive - internal control; b) Invasive micropapillary carcinoma showing weak cytoplasmic staining (Score 1); c) moderate degree of staining (IMPC) (Score 2); d) severe degree of staining (IDC) (Score 3)
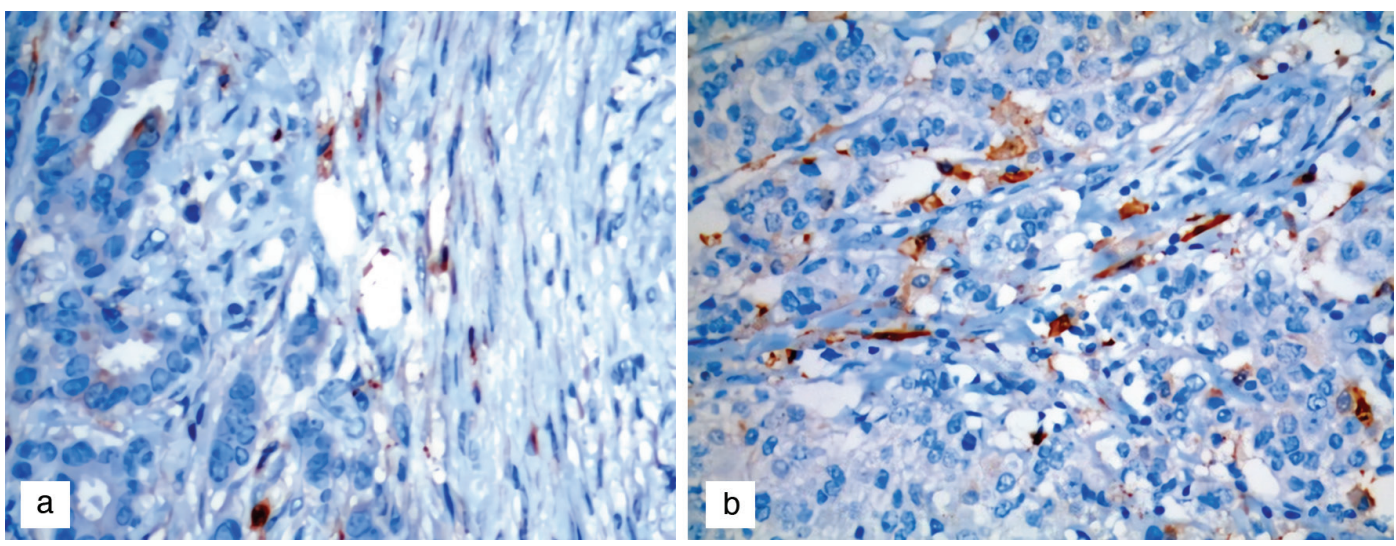

Figure 2. Galectin-3 immunostaining within CAF's, a) weak staining, b) strong staining 
Table 1. Clinicopathologic features of the cases in IMPC and IDC groups

\begin{tabular}{|c|c|c|c|}
\hline & & \multicolumn{2}{|c|}{ Histologic type } \\
\hline & & IMPC (n = 34) & IDC $(n=34)$ \\
\hline \multicolumn{2}{|l|}{ Age (min-max) mean \pm SD } & $(34-80) 56 \pm 11.73$ & (29-80) $54.26 \pm 12.73$ \\
\hline \multirow[t]{2}{*}{ Gender } & Female & 32 & 34 \\
\hline & Male & 2 & 0 \\
\hline \multirow[t]{2}{*}{ Side } & Right & 20 & 18 \\
\hline & Left & 14 & 16 \\
\hline \multirow[t]{2}{*}{ Tumor Galectin-3 } & Negative & 15 & 24 \\
\hline & Positive & 19 & 10 \\
\hline \multirow[t]{2}{*}{ CAF Galectin-3 } & Negative & 10 & 9 \\
\hline & Positive & 24 & 25 \\
\hline \multirow[t]{2}{*}{ Nuclear Grade } & 2 & 11 & 15 \\
\hline & 3 & 23 & 19 \\
\hline \multirow[t]{3}{*}{ Histologic Grade } & 1 & 0 & 1 \\
\hline & 2 & 19 & 19 \\
\hline & 3 & 15 & 14 \\
\hline \multicolumn{2}{|l|}{$\begin{array}{l}\text { Tumor size }(\mathrm{cm}) \\
(\min -\max ) \text { mean } \pm S D\end{array}$} & $(1-8) 2.91 \pm 1.71$ & $(1-6) 2.76 \pm 1.44$ \\
\hline \multirow[t]{3}{*}{ pT stage } & 1 & 12 & 15 \\
\hline & 2 & 18 & 16 \\
\hline & 3 & 4 & 3 \\
\hline \multirow[t]{2}{*}{ Angiolymphatic invasion } & Absent & 10 & 13 \\
\hline & Present & 24 & 21 \\
\hline \multirow[t]{2}{*}{ Perineural invasion } & Absent & 30 & 26 \\
\hline & Present & 4 & 8 \\
\hline \multirow[t]{2}{*}{ Multifocality } & Absent & 26 & 27 \\
\hline & Present & 8 & 7 \\
\hline \multirow[t]{2}{*}{ Multicentricity } & Absent & 28 & 28 \\
\hline & Present & 6 & 6 \\
\hline \multirow[t]{2}{*}{ In situ component } & Absent & 6 & 8 \\
\hline & Present & 28 & 26 \\
\hline \multirow[t]{2}{*}{ Microcalcification } & Absent & 11 & 14 \\
\hline & Present & 23 & 20 \\
\hline \multicolumn{2}{|l|}{$\begin{array}{l}\text { Number of positive nodes } \\
\text { (min-max) mean } \pm \text { SD }\end{array}$} & $(0-20) 5.18 \pm 6.29$ & $(0-33) 6.59 \pm 8.60$ \\
\hline \multirow[t]{4}{*}{ pN stage } & 0 & 8 & 8 \\
\hline & 1 & 11 & 8 \\
\hline & 2 & 6 & 10 \\
\hline & 3 & 8 & 8 \\
\hline \multirow[t]{2}{*}{ Extranodal extension } & Absent & 9 & 10 \\
\hline & Present & 15 & 16 \\
\hline \multicolumn{2}{|l|}{$\begin{array}{l}\text { Ki-67 index }(\%) \\
(\min -\max ) \text { mean } \pm \text { SD }\end{array}$} & (5-90) $30.76 \pm 18.68$ & (5-90) $29.5 \pm 19.94$ \\
\hline
\end{tabular}


Table 1. Continued

\begin{tabular}{|c|c|c|c|}
\hline & & \multicolumn{2}{|c|}{ Histologic type } \\
\hline & & IMPC (n = 34) & IDC $(n=34)$ \\
\hline \multirow[t]{2}{*}{ Ki-67* } & $<20 \%$ & 7 & 8 \\
\hline & $>20 \%$ & 26 & 18 \\
\hline \multirow[t]{2}{*}{ Mutant p53 } & Absent & 15 & 8 \\
\hline & Present & 8 & 0 \\
\hline \multirow[t]{4}{*}{ Molecular subtype } & $A$ & 6 & 6 \\
\hline & B & 22 & 22 \\
\hline & $\mathrm{TN}$ & 2 & 2 \\
\hline & HER2 & 4 & 4 \\
\hline \multirow[t]{2}{*}{ Adjuvant chemotherapy } & Absent & 2 & 3 \\
\hline & Present & 32 & 31 \\
\hline \multirow[t]{2}{*}{ Hormonotherapy } & Absent & 7 & 5 \\
\hline & Present & 27 & 29 \\
\hline \multirow[t]{2}{*}{ Radiotherapy } & Absent & 4 & 6 \\
\hline & Present & 30 & 28 \\
\hline \multirow[t]{2}{*}{ Metastasis } & Absent & 28 & 25 \\
\hline & Present & 6 & 9 \\
\hline DFS & & $(1-105)$ & $(4-113)$ \\
\hline$(\min -\max )$ mean $\pm \mathrm{SD}$ & & $55.09 \pm 24.81$ & $77.5 \pm 33.56$ \\
\hline os & & $(1-105)$ & $(4-113)$ \\
\hline$(\min -\max )$ mean $\pm S D$ & & $61.26 \pm 21.88$ & $87.15 \pm 26.4$ \\
\hline \multirow[t]{2}{*}{ Survival } & Alive & 29 & 25 \\
\hline & Exitus & 5 & 9 \\
\hline
\end{tabular}

Table 2. Association of Galectin-3 expression with histologic type

\begin{tabular}{llccc}
\hline & & IMPC & IDC & P-value \\
\hline \multirow{2}{*}{ Tumor Galectin-3 } & Positive $(n ; \%)$ & $19(55.9 \%)$ & $10(29.4 \%)$ & \\
& Negative $(n ; \%)$ & $15(44.1 \%)$ & $24(70.6 \%)$ & 0.049 \\
\multirow{2}{*}{ CAF Galectin-3 } & Positive $(n ; \%)$ & $24(70.6 \%)$ & $25(73.5 \%)$ & 1
\end{tabular}

IMPC: Invasive micropapillary carcinoma, IDC: Invasive ductal carcinomas, CAF: Cancer-associated fibroblasts, n: Number

Galectin-3 positive staining in tumor cells compared to negative cases, but this difference did not reach statistical significance using Log-Rank tests. However, DFS and OS rates were higher in patients with no Galectin-3 expression in CAFs but once again, this difference did not reach statistical significance.

\section{Discussion and Conclusion}

IMPC of the breast usually exhibits axillary lymph node involvement at the time of diagnosis. In this study, we investigated the levels of
Galectin-3 expression, both in tumor cells and CAFs in IMPCs and sought to ascertain any differences in Galectin-3 expression in IMPCs compared to IDC (non-IMPC) tumors. Accordingly, the level of Galectin-3 expression in invasive tumor cells was significantly higher in the IMPC group than in the IDC group. No significant correlation was found between Galectin-3 expression levels and clinicopathological parameters in the IMPC group. However, loss of Galectin-3 expression in tumor cells in IMPC yielded a result close to significance with larger tumor size and more advanced $\mathrm{pT}$ stages. Then, all cases, regardless of 
Eur J Breast Health 2021; 17(4): 341-351

Table 3. Distribution of clinicopathological parameters in groups of tumor with and without Galectin-3 expression

Tumor Galectin-3 (-)

(n)

Age (mean \pm SD)

Side

Right

Left

Nuclear Grade

2

3

Histologic Grade

1

2

3

Tumor size $(\mathrm{cm})$ (mean \pm SD)

pT stage

1

2

3

Angiolymphatic invasion

Absent

Present

Perineural invasion

Absent

Present

Multifocality

Absent

Present

Multicentrisity

Absent

Present

In situ component

Absent

Present

Microcalcification

Absent

Present

Number of positive nodes

(mean \pm SD)

pN stage

0

1

2

3
$54.82 \pm 13.12$

21

18

14

25

1

21

17

$3.15 \pm 1.83$

13

17

7

14

25

30

31

30

9

9

30

15

24

$7.45 \pm 8.89$

9

7

10

12
Tumor Galectin- 3 (+)

(n)

$55.55 \pm 11.01$

0.81

17

12

0.89

0.83

17

0

17

0.66

12

$2.41 \pm 1.02$

0.038 *

14

15

0

0.046 *

0.87

20

26

3

22

0.95

7

26

3

0.30

0.77

24

10

19

0.93

$.86 \pm 4.65$

0.037 *

7

12

6

0.14 
Table 3. Continued

Tumor Galectin-3 (-)

(n)

\section{Extranodal extension}

Absent

Present

Ki-67 index (\%) (mean \pm SD)

Ki-67

$<20 \%$

$>20 \%$

Mutant p53

Absent

Present

Molecular subtype

A

B

TN

HER2

Adjuvant chemotherapy

Absent

Present

Hormonotherapy

Absent

Present

Radiotherapy

Absent

Present

Metastasis

Absent

Present

\section{Survival}

alive

exitus
11

19

$28.47 \pm 16.58$

Tumor Galectin- 3 (+)

$32.91 \pm 22.60$

2

6

28

1

4

4

35

6

33

8

31

30

9

29

10 (n)

8

12

6

17

9

6

0.18

p-value

1

0.39

1

3

4

1

28

0.55

0.81

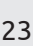

2

27

0.22

23

6

1

25

4

*mutant p53 defines positive nucleer staining in more than $50 \%$ of the invasive tumor cells, and was evaluated in 23 cases with IMPC and 8 cases with IDC. SD: Standard deviation, n: Number, IMPC: Invasive micropapillary carcinoma, IDC: Invasive ductal carcinomas

histological type, were divided into two groups according to whether the tumor cells displayed Galectin-3 expression or not. On analysis in cases with no Galectin-3 expression in tumor cells there was a significant association with larger tumor size, more advanced $\mathrm{T}$ stage, and a greater number of metastatic lymph nodes compared to those with Galectin-3 expression.

Only a few studies have investigated the relationship between Galectin-3 expression with histological type in breast carcinomas (15, 16). In one of these studies, invasive tubular carcinomas and IDC cases in the pT1 stage were compared for immunohistochemical expression of Galectin-3 in tumor cells (15). Widespread cytoplasmic and/or nuclear Galectin-3 immune expression was detected in full-thickness sections of tumors, in invasive tubular carcinomas, compared to histological grade 1 IDCs. In the other study, an H-score was calculated by considering the cytoplasmic and/or nuclear staining in tumor cells in full-thickness sections from 218 cases with IDC and 25 cases with invasive lobular carcinoma (16). The authors reported that Galectin-3 nuclear expression was more common in invasive lobular carcinomas compared to IDC cases. To the best of our knowledge, only a single study has investigated Galectin-3 expression in IMPC as a special histological subtype (17). In this study, the relationship between tumor cells and with the stroma in the pancreas and periampullary region IMPCs was evaluated with immunohistochemical staining for E-cadherin and Galectin-3, and diffuse and strongly intense cytoplasmic staining was detected in invasive tumor cells in all cases. The researchers reported that Galectin-3 may be a marker for tumor 
Eur J Breast Health 2021; 17(4): 341-351

Table 4. Distribution of clinicopathological parameters in CAF Galectin-3 negative and positive groups of tumor

\section{CAF Galectin-3 (-)}

(n)

Age (mean \pm SD)

Side

Right

Left

Nuclear Grade

2

3

Histologic Grade

1

2

3

Tumor size $(\mathrm{cm})$ (mean \pm SD)

pT stage

1

2

3

Angiolymphatic invasion

Absent

Present

Perineural invasion

Absent

Present

Multifocality

Absent

Present

Multicentrisity

Absent

Present

In situ component

Absent

Present

Microcalcification

Absent

Present

Number of positive nodes

(mean \pm SD)

\section{pN stage}

0

1

2

3
$53.26 \pm 13.39$

12

7

7

12

\section{0}

12

7

$2.89 \pm 1.52$

\section{7}

10

2

\section{5}

14

15

4

16

3

17

2

4

15

\section{8}

11

$5.26 \pm 6.67$

8

2

4

5
CAF Galectin-3 (+)

(n)

$55.86 \pm 11.75$

0.43

0.63

23

$$
19
$$

30

1

0.65

27

$2.81 \pm 1.60$

0.85

20

24

25

0.95

18

31

0.60

41

8

0.92

37

12

39

10

0.54

10

39

1

17

32

0.77

$6.15 \pm 7.99$

0.67

8

17

12

0.08 
Table 4. Continued

CAF Galectin-3 (-)

(n)

\section{Extranodal extension}

Absent

Present

Ki-67 index (\%) (mean \pm SD)

Ki-67

$<20 \%$

$>20 \%$

Mutant p53

Absent

Present

Molecular subtype

A

B

TN

HER2

Adjuvant chemotherapy

Absent

Present

Hormonotherapy

Absent

Present

Radiotherapy

Absent

Present

Metastasis

Absent

Present

\section{Survival}

Alive

Exitus

CAF: Cancer-associated fibroblast, SD: Standard deviation, n: Number

progression and metastasis in other pancreatic tumor types, based on Galectin-3 positivity detected in tumor cells in micropapillary carcinoma, a subtype of pancreatic neoplasia with a high grade and high metastatic potential. In our study, Galectin-3 immunostaining in tumor cells was significantly higher in the IMPC group than in the IDC group. However, we did not find a significant relationship between Galectin-3 positivity seen in tumor cells within IMPC and clinicopathological parameters. This suggests that Galectin-3 may play a role in micropapillary morphology, as Galectin-3 has been reported to affect cell surface polarization (18).

There are conflicting findings in studies evaluating the relationship between Galectin-3 expression in tumor cells and clinicopathological parameters in breast carcinomas. In the study of Ilmer et al., among all 87 patients with breast cancer who received chemotherapy and
8

$25.36 \pm 19.71$

6

8

6

1

5

9

2

3

1

18

3

16

5

14

16

3

17

2

CAF Galectin-3 (+)

p-value

(n)

17

23

$31.71 \pm 17.86$

36

0.76

7

2

5

4

45

9

40

1

0.19

44

37

12

0.65

axillary lymph node metastases, lymphovascular invasion was detected less frequently in those with higher Galectin-3 expression (H-score level $\geq 150$ ). However, no relationship was found between Galectin-3 expression level and age, ER/PR/CerbB2 expression status, the number of positive lymph nodes, stage, and histological grade (19). In the study of Zhang et al., examining positive cytoplasmic and/or nuclear Galectin-3 expression in full-thickness sections containing tumor, increased Galectin-3 expression was correlated with young age, increased tumor size, higher histological grade, a greater number of metastatic lymph nodes, and triple-negative molecular subtype (13). In our study, contrary to this finding, we found larger tumor size, more advanced $\mathrm{T}$ stage, and a greater number of metastatic lymph nodes in cases where Galectin-3 expression was not detected in tumor cells. However, other studies have not detected a relationship between tumor 
Table 5. Analysis of the cases in terms of distant metastasis and survival according to Galectin-3 expression status

\begin{tabular}{|c|c|c|c|c|c|}
\hline & & \multicolumn{2}{|c|}{ Tumor Galectin-3 } & \multicolumn{2}{|c|}{ CAF Galectin-3 } \\
\hline & & $\begin{array}{c}(-) \\
n / \%\end{array}$ & $\begin{array}{c}(+) \\
n / \%\end{array}$ & $\begin{array}{c}(-) \\
n / \%\end{array}$ & $\begin{array}{c}(+) \\
n / \%\end{array}$ \\
\hline \multicolumn{2}{|c|}{ Exitus cases $*(n=14 ; 20.6 \%)$} & $10(71.4 \%)$ & $4(28.6 \%)$ & $2(14.2 \%)$ & $12(85.8 \%)$ \\
\hline \multicolumn{2}{|c|}{ Median time, month (exitus cases*) } & 42.5 & 63.5 & 41.5 & 54 \\
\hline \multicolumn{2}{|c|}{ Metastatic cases ( $n=15 ; 22.1 \%$ ) } & $9(60 \%)$ & $6(40 \%)$ & $3(20 \%)$ & $12(80 \%)$ \\
\hline \multicolumn{2}{|c|}{ Median time, month (metastasis) } & 34 & 36 & 65 & 32.5 \\
\hline \multirow{6}{*}{ Metastasis site** } & Bone & 7 & - & - & 7 \\
\hline & Brain & 2 & 2 & 1 & 3 \\
\hline & Lung & 1 & 2 & - & 3 \\
\hline & Liver & 2 & 1 & 1 & 2 \\
\hline & Neck lymph nodes & - & 1 & - & 1 \\
\hline & Mediastinum & 1 & - & 1 & - \\
\hline
\end{tabular}

size, T-stage and Galectin-3 expression $(16,20)$. In another study that included 116 breast cancer patients where staining intensity of $\geq 30 \%$ in tumor cells was considered as Galectin-3 positivity, Galectin-3 positivity was associated with increased lymphovascular invasion and a higher rate of PR expression (20). Many studies in the literature have investigated the relationship between Galectin-3 expression in tumor cells and prognosis. Some have found an association between Galectin-3 expression levels in tumor cells and good $(19,20)$ or poor prognosis (21), while others were unable to detect any correlation between Galectin-3 expression levels in tumor cells and prognosis (13, $16,22)$. In a study, conducted with a large patient population ( $\mathrm{n}=$ 1086), immunhistochemical expression of Galectin-3 in tumor cells was not associated with survival, while a significant correlation was found with drug resistance. These authors suggested that treatment models targeted at Galectin-3 may be useful in preventing resistance to chemotherapeutic drugs (13).

A limited number of studies have evaluated the relationship between the expression of Galectin-3 in stroma and the clinicopathological parameters in breast carcinomas. In a study involving 273 breast cancer patients, higher histological grade, and more advanced $\mathrm{pN}$ stage were reported in patients with Galectin-3 expression in stromal fibroblasts (16). In the same study, cases with Galectin-3 expression in stromal fibroblasts were found to be associated with worse prognosis, whereas no significant relationship between Galectin-3 expression in tumor cells and survival rates was detected (16). Logullo et al. examined the immunhistochemical expression of Galectin-3 in tumor cells and stromal fibroblasts in 92 early-stage breast carcinoma cases (22). In approximately half of the cases, tumor cells stained positively with Galectin-3 (cytoplasmic staining), while in more than half of them staining of the stromal (nuclear quality) component was observed. These authors speculated that the intracellular localization of Galectin-3 may vary, depending on the tumorigenicity of the cell. In the same study, the immune expression of Galectin-3 in tumor cells or stroma was not associated with DFS and OS. However, it was not explicitly stated which cellular component in the stroma was stained in the level of Galectin-3 expressed in CAFs between a histological subtype of IMPC cases and IDC cases. In addition, considering all the cases, regardless of histological type, we did not find a significant difference in terms of DFS and OS between patients with and without CAF Galectin-3 expression. However, cases with distant metastasis and/or patients who had died showed higher rates of Galectin-3 positivity in CAFs.

Some experimental studies have suggested that the loss of Galectin-3 expression in tumor cells increased the metastatic potential of the tumor to regions such as lymph nodes and bone marrow (23), while decreased Galectin-3 expression in stroma affected the adhesion molecules in the tumor microenvironment and increased the metastatic potential of tumor cells (24). However, to our knowledge, no study has investigated the relationship between Galectin-3 expression and the region of metastasis in breast carcinomas in humans. In the current study, in the cases with distant metastases, tumor cells were negative for Galectin-3 in $60 \%$ of the cases, while CAFs were positive for Galectin-3 in $80 \%$ of the cases. Similarly, in patients who had died from breast cancer, tumor cells were negative for Galectin-3 in $71.4 \%$ while CAFs were positive for Galectin-3 in $85.8 \%$. Bone was the most common site for metastasis in our study. Indeed, in all cases with bone metastasis ( $n=7)$, Galectin-3 expression was absent in the tumor cells, but present in the CAFs.

Our study compared a specific histological type (IMPC) with IDC patients in terms of immunhistochemical expression of Galectin-3 in a limited number of patient populations who underwent a median of 79 months clinical follow-up. The limitations of this study are the low number of patients and the low level of $\mathrm{H}$-score that was considered positive for Galectin-3 in tumor cells.

In this study, loss of Galectin-3 expression in tumor cells was found to be associated with aggressive clinical parameters, including larger tumor size, advanced pT stage and a higher number of metastatic nodes, and this relationship appeared to be independent of the histological tumor type. 


\section{References}

1. Fisher ER, Palekar AS, Redmond C, Barton B, Fisher B. Pathologic findings from the National Surgical Adjuvant Breast Project (protocol no. 4). VI. Invasive papillary cancer. Am J Clin Pathol 1980; 73: 313-322. (PMID:7361710) [Crossref]

2. Siriaunkgul S, Tavassoli FA. Invasive micropapillary carcinoma of the breast. Mod Pathol 1993; 6: 660-662. (PMID:8302807) [Crossref]

3. Nassar H. Carcinomas with micropapillary morphology: clinical significance and current concepts. Adv Anat Pathol 2004; 11: 297-303. (PMID:15505530) [Crossref]

4. Nassar H, Pansare V, Zhang H, Che M, Sakr W, Ali-Fehmi R, et al. Pathogenesis of invasive micropapillary carcinoma: role of MUC1 glycoprotein. Mod Pathol 2004; 17: 1045-1050. (PMID:15154007) [Crossref]

5. Yang Y-L, Liu B-B, Zhang X, Fu L. Invasive micropapillary carcinoma of the breast: an update. Arch Pathol Lab Med 2016; 140: 799-805. (PMID:27472238) [Crossref]

6. Luna-Moré S, Gonzalez B, Acedo C, Rodrigo I, Luna C. Invasive micropapillary carcinoma of the breast. A new special type of invasive mammary carcinoma. Pathol Res Pract 1994; 190: 668-674. (PMID:7808965) [Crossref]

7. Spano D, Heck C, De Antonellis P, Christofori G, Zollo M. Molecular networks that regulate cancer metastasis. Semin Cancer Biol 2012; 22: 234-249. (PMID:22484561) [Crossref]

8. De Vlieghere E, Verset L, Demetter P, Bracke M, De Wever O. Cancerassociated fibroblasts as target and tool in cancer therapeutics and diagnostics. Virchows Arch 2015; 467: 367-382. (PMID:26259962) [Crossref]

9. Fortuna-Costa A, Gomes AM, Kozlowski EO, Stelling MP, PavÃ£o MSG. Extracellular galectin-3 in tumor progression and metastasis. Front Oncol 2014; 4: 138 (PMID:24982845) [Crossref]

10. Ruvolo PP. Galectin 3 as a guardian of the tumor microenvironment. Biochim Biophys Acta 2016; 1863: 427-437. (PMID:26264495) [Crossref]

11. Elola MT, Ferragut F, Méndez-Huergo SP, Croci DO, Bracalente C, Rabinovich GA. Galectins: Multitask signaling molecules linking fibroblast, endothelial and immune cell programs in the tumor microenvironment. Cell Immunol 2018; 333: 34-45. (PMID:29602445) [Crossref]

12. Farhad M, Rolig AS, Redmond WL. The role of Galectin-3 in modulating tumor growth and immunosuppression within the tumor microenvironment. OncoImmunology 2018; 7: e1434467. (PMID:29872573) [Crossref]

13. Zhang H, Luo M, Liang X, Wang D, Gu X, Duan C, et al. Galectin-3 as a marker and potential therapeutic target in breast cancer. PLoS One 2014; 9: e103482. (PMID: 25254965) [Crossref]
14. Romero A, Gabius H-J. Galectin-3: is this member of a large family of multifunctional lectins (already) a therapeutic target? Expert Opin Ther Targets 2019; 23: 819-828. (PMID:31575307) [Crossref]

15. Fernandez-Aguilar S, Noël J-C. Expression of cathepsin D and galectin 3 in tubular carcinomas of the breast. APMIS 2008; 116: 33-40. (PMID:18254778) [Crossref]

16. Moisa A, Fritz P, Eck A, Wehner H-D, Mürdter T, Simon W, et al. Growth/Adhesion-regulatory tissue lectin galectin-3: stromal presence but not cytoplasmic/nuclear expression in tumor cells as a negative prognostic factor in breast cancer. Anticancer Res. 2007; 27: 2131-2139. (PMID:17695496) [Crossref]

17. Khayyata S, Basturk O, Adsay NV. Invasive micropapillary carcinomas of the ampullo-pancreatobiliary region and their association with tumor-infiltrating neutrophils. Mod Pathol 2005; 18: 1504-1511. (PMID:16007065) [Crossref]

18. Piyush T, Chacko AR, Sindrewicz P, Hilkens J, Rhodes JM, Yu L-G. Interaction of galectin-3 with MUC1 on cell surface promotes EGFR dimerization and activation in human epithelial cancer cells. Cell Death Differ 2017; 24: 1937-1947. (PMID:28731466) [Crossref]

19. Ilmer M, Mazurek N, Gilcrease MZ, Byrd JC, Woodward WA, Buchholz TA, et al. Low expression of galectin-3 is associated with poor survival in node-positive breast cancers and mesenchymal phenotype in breast cancer stem cells. Breast Cancer Res 2016; 18: 97. (PMID:27687248) [Crossref]

20. Yamaki S, Fujii T, Yajima R, Hirakata T, Yamaguchi S, Fujisawa T, et al. Clinicopathological significance of decreased galectin-3 expression and the long-term prognosis in patients with breast cancer. Surg Today 2013; 43: 901-905. (PMID:23090139) [Crossref]

21. Jones C, Mackay A, Grigoriadis A, Cossu A, Reis-Filho JS, Fulford L, et al. Expression profiling of purified normal human luminal and myoepithelial breast cells: identification of novel prognostic markers for breast cancer. Cancer Res 2004; 64: 3037-3045. (PMID:15126339) [Crossref]

22. Logullo AF, Lopes ABG, Nonogaki S, Soares FA, Netto MM, Nishimoto IN, et al. C-erbB-2 expression is a better predictor for survival than galectin-3 or p53 in early-stage breast cancer. Oncol Rep 2007; 18: 121126. (PMID:17549356) [Crossref]

23. Pereira JX, dos Santos SN, Pereira TC, Cabanel M, Chammas R, de Oliveira FL, et al. Galectin-3 regulates the expression of tumor glycosaminoglycans and increases the metastatic potential of breast cancer. J Oncol 2019; 2019: 1-15. (PMID:31949431) [Crossref]

24. Pereira JX, Azeredo MCB, Martins FS, Chammas R, Oliveira FL, Santos $\mathrm{SN}$, et al. The deficiency of galectin-3 in stromal cells leads to enhanced tumor growth and bone marrow metastasis. BMC Cancer 2016; 16: 636 . (PMID:27526676) [Crossref] 